\title{
APUNTES SOBRE EL ARTE DE LA NEGOCIACIÓN: INTERCULTURALIDAD Y CORTESÍA EN EL INGLÉS EMPRESARIAL
}

\author{
J.J. Lanero. Universidad de León
}

\begin{abstract}
A pesar de que la enseñanza de la negociación intercultural ya viene ocupándose de las diferencias culturales en la estrategia negociadora, poca importancia se ha dado a los efectos de las diversas estrategias de cortesía. Estudios recientes revelan que la cortesía es importante porque representa mucho, y muy distinto, en los entornos culturales mixtos y habituales en el mundo internacional de los negocios. El presente trabajo considera esas diferencias e indica que estar bien informado sobre las diferencias culturales en la cortesía debe formar parte de la educación del futuro negociador.
\end{abstract}

\section{INTRODUCCIÓN}

Aunque la enseñanza de la negociación intercultural se ha ocupado de las diferencias culturales a la hora de plantear una estrategia negociadora, poca, cuando no nula, atención se ha prestado a los efectos de la utilización de diferentes estrategias de cortesía.

En la actualidad existe un conjunto importante de investigaciones que tiene sus raíces en la Teoría de la Cortesía expuesta por Brown y Levinson (1987), que revelan que la cortesía es importante porque representa diferentes aspectos en entornos culturales mixtos, típicos en el mundo internacional de la empresa.

Las líneas que siguen consideran algunas de estas diferencias y sugieren que estar bien informados sobre las diferencias culturales en la cortesía debe constituir una destreza más del futuro profesional de los negocios y, consecuentemente, pasar a ser un elemento importante en el aprendizaje del inglés empresarial como lengua franca en las transacciones comerciales.

La cortesía es importante en los escenarios interculturales y, por supuesto, en las negociaciones de empresa. No obstante, lo que es cortesía varía de cultura a cultura y las negociaciones pueden fracasar si se utiliza mal o se malinterpretan las estrategias de cortesía. A pesar de que las investigaciones que se han hecho en el campo de la negociación han dedicado especial atención a las diversas estrategias que se pueden emplear y a las diferencias culturales (Morgan and Stripp, 1991), en comparación, poca atención se ha prestado al papel que desempeña la cortesía. La situación, empero, está cambiando y los trabajos en la floreciente 
literatura académica sobre comunicación y negociación (Firth, 1994; Bargiela-Chiappini and Harris, 1997; Neimeir, Campbell and Dirven, 1998) están centrando su interés en la cortesía, sobre todo dentro de la teoría desarrollada por Brown y Levinson (1987), que nosotros también utilizamos como punto de referencia en este trabajo.

\section{DEFINICIÓN DEL PROCESO NEGOCIADOR}

La negociación se produce cuando existe una diferencia o incompatibilidad entre dos o más partes (Julian, 1990).

Joyce Neu (1985) apoyándose en el artículo "Models of the interaction of language and social life" (Hymes, 1972: 35-71) caracteriza el lenguaje de la negociación según se muestra en el Cuadro 1.

\section{Cuadro 1}

Rasgos distintivos del lenguaje en la negociación

\begin{tabular}{|l|l|}
\hline Participantes: & $\begin{array}{l}\text { 1. Existe un mínimo de dos partes que están presentes. } \\
\text { 2. Ambas partes tienen objetivos predeterminados. } \\
\text { 3. Alguno de los objetivos predeterminados no es compartido por ambas } \\
\text { partes. }\end{array}$ \\
\hline Fines: & $\begin{array}{l}\text { 1. Existe un resultado. } \\
\text { 2. Ambas partes creen que el resultado de la negociación puede ser } \\
\text { satisfactorio. }\end{array}$ \\
\hline Hechos: & $\begin{array}{l}\text { 1. Ambas partes desean modificar su postura. } \\
\text { 2. Los postulados incompatibles de las partes dificultan la modificación de las } \\
\text { posturas. }\end{array}$ \\
\hline Normas: & 1. Las partes entienden el propósito de la negociación. \\
\hline
\end{tabular}

Adaptado de Neu (1985: 38)

Lograr un resultado es lo que conlleva todo proceso de negociación. Cualquier encuentro intercultural es un ejemplo de negociación de comprensión mutua y, en un escenario intercultural, alcanzar ese entendimiento puede ser especialmente problemático debido a las diferencias entre las partes involucradas en lo que se refiere a normas y expectativas. Este extremo está muy bien documentado en una negociación de ventas real analizada por Helen E. Marriot (1990: 33-65), en donde las partes involucradas resultaron estar obrando con propósitos opuestos. Mientras que el vendedor australiano asumía que la finalidad de la reunión era iniciar una venta, el potencial comprador la consideraba una oportunidad para obtener información que transmitiría más tarde a su oficina central en Japón. Resultado final: una considerable cantidad de malos entendidos y frustraciones.

Las diferencias normativas también encuentran eco en el comportamiento y estilo de comunicación que se consideran efectivos y apropiados dentro del transcurso del encuentro. Por lo general, los negociadores angloamericanos se comportan según las normas del Sistema de Discurso Utilitario (Scollon and Scollon, 1995), que valora la franqueza, la claridad y la igualdad de oportunidades para hablar, mientras que los negociadores japoneses y chinos provienen de una tradición cultural que tiene sus raíces en los valores defendidos por Confucio, en los que el silencio significa atención respetuosa (Bilbow, 1997: 21-48).

Los negociadores angloamericanos demuestran su interés con respuestas, lo que podría considerarse como interrupciones por sus colegas. A su vez, los occidentales pueden interpretar 
el silencio de chinos y japoneses de forma negativa, como falta de interés y franqueza. Todo esto, en su conjunto, arroja un resultado final de malos entendidos y apreciaciones erróneas.

Con independencia de cómo se enfoque la negociación, cada una de las partes estará representado constantemente escenas en las que la expresión de su semblante procurará asegurar un proceso negociador relajado. Ambas partes necesitarán tener en cuenta la reacción que se refleja en la cara del otro y disponer de formas de reconducir la conversión con el fin de lograr una conclusión satisfactoria y que la relación empresarial prospere.

\section{LA CORTESÍA FACIAL}

El concepto facial es esencial en la Teoría de la Cortesía de Brown y Levinson (1987). Aunque ha sido criticada por ser demasiado europea, esta teoría nos da un conjunto importante de categorías para estudiar y analizar la cortesía. Definen el rostro como

... the public self-image that every member wants to claim for himself... . Face is something that is emotionally invested, and can be lost, maintained or enhanced, and must be constantly attended to in interaction. In general people cooperate (and assume each other's cooperation) in maintaining face in interaction, such cooperation being based on mutual vulnerability of face. (Brown and Levinson, 1987: 61)

Algunos estudiosos japoneses y chinos han ofrecido una versión alternativa de la expresión de la cara; la consideran "algo" que la sociedad concede al individuo para que la conserve impoluta en beneficio de la colectividad y no de sí mismo. Así, mientras que el individualismo de la cultura angloamericana fomenta el "auto-diseño" de la expresión y semblante faciales, en las culturas japonesa y china la gente se ve obligada a proteger su cara de los que la rodean (Bilbow, 1997: 43).

Brown y Levinson (1987) proponen dos clases de semblante y cortesía: positivo y negativo. Un rostro positivo está relacionado con ser sociable y solidario; mientras que el negativo tiene que ver con la libertad de acción y con la recepción y expresión de deferencia. Las personas tienen que reaccionar constantemente ante la expresión facial de los demás. Por una parte, el deseo de ser sociable y, por la otra, la necesidad de libertad e independencia.

\begin{tabular}{|c|c|}
\hline \begin{tabular}{|l} 
Rostro positivo \\
\end{tabular} & Rostro negativo \\
\hline 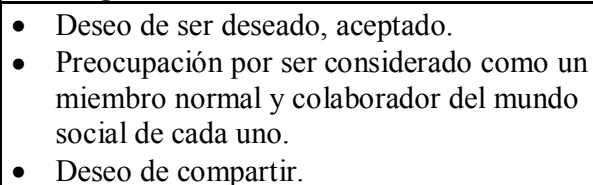 & $\begin{array}{l}\text { - Deseo de que los demás no pongan trabas. } \\
\text { - Conservar el derecho a la independencia, la } \\
\text { libertad de acción dentro del territorio de } \\
\text { cada uno. } \\
\text { - Derecho a no ser molestado. }\end{array}$ \\
\hline
\end{tabular}

Conseguir y conservar estos dos tipos de cortesía implica utilizar estrategias de cortesía positivas y negativas o estrategias solidarias y de deferencia, si nos ajustamos a la terminología propuesta por algunos autores (Scollon and Scollon, 1995).

Según Brown y Levinson (1987) la elección de la estrategia de cortesía viene determinada por consideraciones de poder, distancia y grado de imposición.

Poder significa hasta qué punto una persona tiene autoridad sobre otra como para influir en sus acciones.

Distancia es sinónimo de la cercanía o lejanía que se pude derivar de la frecuencia de los contactos.

Odisea, $n^{0} 1,2001$ 
La imposición se refiere al nivel de esfuerzo que un individuo tiene que realizar, ya sea físico o psíquico, para hacer o rechazar hacer algo.

Con el fin de evitar cualquier tipo de imposición, los hablantes intentan:

- minimizar el coste a la otra persona.

- maximizar el beneficio a la otra persona.

Los hablantes variarán su grado de diplomacia dependiendo de:

- cómo perciban el beneficio-coste para el que escucha.

- los derechos del hablante en relación con su interlocutor.

Los conceptos de beneficio-coste y los derechos del hablante oscilarán de una cultura a otra. En una cultura en la que la idea de poder-distancia es alta (Hofstede, 1984) el hablante acepta ser directo cuando se dirige a un subordinado, pues al hablante se le reconoce ese derecho; mientras que en una cultura en la que la idea de poder-distancia es baja, el hablante no dispondrá de ese derecho. En semejante contexto cultural las convencionales órdenes y peticiones indirectas serán la norma a seguir. Robert March recoge en su obra, Working for a Japanese Company, cómo un americano repara que, a la hora de plantear peticiones, “... we ... feel that we have to preface our remarks with 'I'd appreciate it if you would"' (March, 1992: $100)$.

\begin{tabular}{|l|l|}
\hline $\begin{array}{l}\text { Estrategias de cortesía solidaria } \\
\text { (cortesía positiva) }\end{array}$ & $\begin{array}{l}\text { Estrategias de cortesía de deferencia } \\
\text { (cortesía negativa) }\end{array}$ \\
\hline $\begin{array}{l}\text { La importancia de una sintonía entre las } \\
\text { partes. }\end{array}$ & $\begin{array}{l}\text { La importancia de mantener distancias entre } \\
\text { los participantes. }\end{array}$ \\
$\begin{array}{l}\text { Asumir que hay poca distancia entre los } \\
\text { participantes y que las diferencias de poder } \\
\text { entre ambos son pequeñas. }\end{array}$ & $\begin{array}{l}\text { El hablante, sin respetar la expresión facial } \\
\text { negativa del interlocutor, avanza } \\
\text { cuidadosamente en su imposición y procura } \\
\text { dar al que escucha una salida en el caso de } \\
\text { que éste considere exagerada la imposición. }\end{array}$ \\
$\begin{array}{l}\text { Ejemplos de comportamiento pueden ser: } \\
\text { - Autodominio. }\end{array}$ & $\begin{array}{l}\text { Ejemplos de comportamiento pueden ser: } \\
\text { - Cortesía solidaria. }\end{array}$ \\
\hline
\end{tabular}

\section{EL PODER SEMÁNTICO}

El denominado poder semántico se pone en funcionamiento cuando existe una diferencia de poder entre el hablante y el destinatario de su mensaje. Esta diferencia se puede ocultar de diversas formas. Una de ellas consiste en utilizar el pronombre de primera persona del plural, we, con el fin de dar sensación de cercanía y solidaridad y no de distanciamiento. En ciertos casos la referencia puede ser ambigua: incluyente y excluyente.

Desafortunadamente, la ambigüedad potencial del we puede llevar a los negociadores a hablar con fines contrapuestos porque, por tradición cultural, están predispuestos a atribuir diferentes significados a la forma de utilizar el pronombre we. Por ejemplo, si la parte B proviene de lo que algunos autores denominan cultura fuertemente entretejida (Simons, Vazques and Harris, 1993), lo que ocasiona claras distinciones entre lo interno y externo, we puede utilizarse para marcar diferencia y exclusión, mientras que si la parte A proviene de lo que se ha acuñado como cultura de tejido flojo, en la que la delimitación del grupo es más permeable, we se usará como un modo de invitar a ser socios. 
Este extremo puede ilustrarse con un párrafo extraído de una simulación de negociación en la que el uso que hace el vendedor del we va de lo excluyente a lo incluyente en la fase inicial de la negociación, cuando se hace la distinción entre socios del grupo internos y externos. Al mismo tiempo se intenta establecer un punto de convergencia que conduzca a un uso incluyente en el que se alude a una experiencia compartida en el pasado como forma de invitar a la cooperación:

Yeah first of all I want to mention that I, our and my Company, we are very happy that you are going to buy these electronic products from our company, Alpha Appliance Distributors, and I think you will be happy as you have been in the past with our products and I think, with the price, it's not only in your company that you have a crisis we have, in our company we have a crisis too and you know the trusts force $u s$ to pay higher wages and therefore I can't sell you these products for nothing, for no money but I'm sure as we did in the past we will come to an agreement.

Los hablantes también pueden utilizar we para mitigar las instrucciones $\mathrm{u}$ órdenes, evitando así la referencia directa al oyente que se produciría si se sirvieran del you o un imperativo no modificado. Algún autor (Bilbow, 1997) ha demostrado cómo en las reuniones multiculturales que se celebran en países asiáticos, los occidentales tienden a utilizar we de la siguiente manera: We need to do that equivale a You need to do that.

Los nativos asiáticos utilizan we para referirse a los presentes en la reunión, lo que es también indicativo de su orientación en comparación con sus colegas occidentales.

\section{ESTRATEGIAS PERSUASIVAS}

La demostración de interés por la otra parte y asegurar la buena calidad del producto y lo satisfactorio que uno puede estar con él son buenas estrategias de persuasión. No obstante, pueden llevar a malos entendidos si un vendedor hace afirmaciones del siguiente cariz:

a) I am sure you will start buying after this first hundred.

b) I am sure as we did in the past we will come to an agreement.

c) I am sure you will be satisfied with our service and cost as well.

En estos ejemplos el vendedor hace juicios de valor por lo que se refiere a acciones y reacciones propias del comprador sobre las que, sin duda, los vendedores tienen poco o ningún control. Haciendo semejantes imputaciones, el vendedor parece ejercer una estrategia de cortesía de deferencia: realiza mínimas concesiones a los deseos del interlocutor o ignora lo que es relevante para él. El resultado que se obtiene es que el efecto que causa en el interlocutor puede ser el de imponerse en su libertad de acción, aunque, casi con toda seguridad, no es lo que el vendedor desea conseguir.

Otras estrategias de deferencia pueden ser pedir disculpas y ser pesimistas, lo que se puede expresar a través de afirmaciones como "I am afraid X". Aunque estas estrategias observan deferencia hacia la otra parte, también crean una distancia entre ambas, dificultando continuar con estrategias solidarias como la búsqueda de un punto de vista en común, opiniones, actitudes, conocimiento, empatía, etc.; ser optimista y asumir o reafirmar reciprocidad.

El uso de tales estrategias de cortesía solidaria puede llevar a un mecanismo de vuelo de persecución cuando hay diferencias culturales en la actitud ante la solidaridad y la deferencia entre gente de status superior e inferior. Si una de las partes obra basándose en la deferencia con la otra parte, el intento solidario puede ser rechazado, con lo que lo que pretendía ser 
colaboración y ayuda se puede percibir como expresión de poder. Es paradójico que el esfuerzo que una parte realiza para reducir la diferencia de poder termine siendo una posición dominante. Es probable que esto sea lo que ocurre en muchos casos entre hablantes nativos de inglés y los que no lo son, sobre todo cuando los primeros procuran poner a sus interlocutores en una situación cómoda empleando estrategias de cortesía solidarias que, en realidad, sólo consiguen que se sientan incómodos.

\section{HACIA UNA ENSEÑANZA DE COMUNICACIÓN INTERCULTURAL}

La solución más sencilla sería enseñar diferentes formas de expresar estrategias de cortesía. Este enfoque sólo abordaría el tema de forma superficial. No obstante, dado que las diferencias entre negociadores no sólo se circunscriben a la forma lingüística, sino también a las diferentes formas de evaluar y utilizar la cortesía, ambas partes necesitan tener presente la orientación que subyace en la otra parte y cómo se sustancia en los diferentes usos que se pueden hacer de las estrategias de cortesía. Hay que poner en duda que cada una de las partes haya sido informada del estilo de cortesía que la otra practica, pues adaptarse en exceso a las formas del interlocutor puede percibirse como una indiscreción que puede crear suspicacias y hasta rechazo (Francis, 1991: 403-428).

En realidad, ambas partes deben adaptarse y es necesario el desarrollo de una destreza en la comunicación intercultural a fin de conceder cierta importancia a las formas que emplean los negociadores de las diferentes culturas y cómo las interpretan.

La cortesía es importante por los distintos aspectos que inciden en los participantes en una negociación intercultural. El desarrollo y comprensión de esas diferencias deberían formar parte del conocimiento de todo negociador instruido de manera integral. Las formas lingüísticas son, fuera de toda duda, indispensables, pero no lo es menos la consciencia cultural. Para que una negociación tenga éxito, se han de conocer el habla, los modos y las costumbres culturales del interlocutor.

Y qué decir del papel que la traducción debería desempeñar. Lo que es correcto, en tanto expresión, en un ámbito cultural, trasladado a otro cobra tintes de superioridad o sumisión, de arrogancia o humillación; todas ellas constituyen obstáculos que dificultarán o impedirán el éxito final. De ahí la importancia de introducir elementos culturales en la formación de los futuros negociadores. De lo contrario habremos conseguido que, aunque de expresión lingüística correcta, sean incorrectos en la observancia de una cortesía intercultural que debe tener punto de equilibrio entre la solidaridad y la deferencia.

El propio Shakespeare debió tener en cuenta que existe algo más que la palabra para negociar, cuando escribió: "Let every eye negotiate for itself, / And trust no agent" (Shakespeare, 1600: II, i, 187).

\section{BIBLIOGRAFÍA}

Bargiela-Chiappini, F. and S. Harris, eds. 1997. The Languages of Business: An International Perspective. Edinburgh University Press.

Bilbow, G.T. 1997. "Spoken discourse in the multicultural workplace in Hong Kong: applying a model of discourse as impression management". The Languages of Business: An International Perspective. Eds. F. Bargiela-Chiappini, and S. Harris. Edinburgh University Press. 21-48. 
Brown, P. and S.C. Levinson. 1987. Politeness: Some Universals in Language Use. Cambridge University Press.

Firth, A., ed. 1994. The Discourse of Negotiation: Studies of Language in the Workplace. Oxford: Elsevier Science.

Francis, J. N.P. 1991. "When in Rome? The effects of cultural adaptation on intercultural business negotiations". Journal of International Business Studies (3 ${ }^{\text {rd }}$ Quarter): 403-428.

Gumperz, J. and D. Hymes, eds. 1972. Directions in Sociolinguistics: the Ethnography of Communication. New York: Holt, Rinehart \& Winston.

Hofstede, G. 1984. Culture's Consequences: International Differences in WorkRelated Values. Beverly Hills, CA: Sage Publications.

Hymes, D. 1972. "Models of the interaction of language and social life". Directions in Sociolinguistics: the Ethnography of Communication. New. Eds. J. Gumperz, and D. Hymes. New York: Holt, Rinehart \& Winston. 35-71.

Julian, M. A. 1990. "Talks: what negotiators think drives a hard bargain". Edinburgh Working Papers in Applied Linguistics. Ed. T. Lynch, $\mathrm{n}^{\mathrm{o}} 1$.

March, R. B. 1992. Working for a Japanese Company. Tokyo \& New York, London: Kodanska.

Marriot, H. E. 1990. Intercultural Business Negotiations: The Problem of Norm Discrepancy. ARAL, Series S. 7: 33-65.

Morgan, R. T. and W. G. Stripp. 1991. Successful International Business Negotiations. Houston: Gulf Publishing Company.

Neimeir, S; C. P. Campbell and R. Dirven, eds. 1998. The Cultural Context in Business Communication. Amsterdam and Philadelphia: John Benjamins.

Neu, J. 1985. A Multivariate Sociolinguistic Analysis of the Speech Event Negotiation. Unpublished Ph. D., University of Southern California.

Scollon, R. and S. W. Scollon. 1995. Intercultural Communication - A Discourse Approach. Oxford: Blackwell.

Shakespeare, William. 1600. Much Ado About Nothing. Act II, Scene i, line 187.

Simons, G. F.; C. Vazques and P.R. Harris. 1993. Transcultural Leadership: Empowering the Diverse Workforce. Houston: Gulf. 